Tôhoku Math. Journ.

20 (1968), 417-424.

\title{
GROWTH CONDITIONS AND THE NUMERICAL RANGE IN A BANACH ALGEBRA
}

\author{
J. G. StAMPFli AND J. P. Williams*)
}

(Received January 22, 1968)

0. Introduction. In this paper, we consider the numerical range in an arbitrary Banach algebra with identity, and study its relation to various growth conditions on the resolvent.

In $\S 1$, we list several facts about this generalized numerical range. Some of these are more or less well known for concrete algebras, but do not seem to have been formulated in their proper generality. In particular, we recognize that part of this section is implicit or explicit in Lumer [7]. Consequently, we have included proofs here only when a result is new (or requires a new technique), or when the proof represents a substantial simplification.

The main result of $\S 2$ is a Phragmén-Lindelöf theorem for quasi-nilpotent elements in a Banach algebra. This yields a sharper version of a similar theorem of Lumer and Phillips [8].

Finally, in $\$ 3$ we apply the methods of $\$ 1$ to study the numerical range in its usual setting, Hilbert space. The notion of essential numerical range appears naturally here, and this set is shown to be chracterized in the way one would expect by analogy with the essential spectrum.

1. Let $\mathcal{A}$ be a complex Banach algebra with unit, and let $\mathfrak{p}=\left\{f \in \mathcal{A}^{*}\right.$ : $f(1)=1=\|f\|\}$ be the set of positive linear functionals on $\mathcal{A}$. (These are called normalized states in [7].) For $x \in \mathcal{A}$, define the numerical range $W_{0}(x)$ as

$$
W_{0}(x)=\{f(x): f \in \mathfrak{p}\}
$$

and the numerical radius $\left|W_{0}(x)\right|$ as

$$
\left|W_{0}(x)\right|=\sup \left\{|z|: z \in W_{0}(x)\right\} .
$$

The results of $\S 1$ are concerned with the relation between $W_{0}(x)$ and first

*) The first author gratefully acknowledges the support of the National Science Foundation. 
order growth conditions on the resolvent of $x$. The terminology is justified by Theorems 1 and 6 . We will use [9] as a , reference for the basic facts about Banach algebras.

THEOREM 1. $W_{0}(x)$ is convex, closed and contains the spectrum $\sigma(x)$ for $x \in \mathcal{A}$.

PROOF. $\mathfrak{p}$ is evidently convex and weak ${ }^{*}$ compact in $\mathcal{A}^{*}$. Since $f \rightarrow f(x)$ is weak ${ }^{*}$ continuous on $\mathscr{A}^{*}$, it follows that the range of this map $\left(=W_{0}(x)\right)$ is compact and convex.

To prove the last assertion, let $\mathcal{C}$ be a maximal commutative subalgebra of $\mathcal{A}$ containing 1 and $x$. Then $o(x)$ is the spectrum of $x$ in the algebra $\mathcal{C}$, and since $\mathcal{C}$ is commutative, $\sigma(x)$ is the set of numbers $\phi(x)$ where $\phi$ ranges over the complex homomorphisms of $\mathcal{C}$. Now, each such $\phi$ can be extended to a positive linear functional $f$ on $\mathcal{A}$, hence, $\phi(x)=f(x) \in W_{0}(x)$.

LEMMA 1. For $\lambda \notin W_{0}(x),\left\|(x-\lambda)^{-1}\right\| \leqq d\left[\lambda, W_{0}(x)\right]^{-1}$.

PROOF. If $\lambda \notin W_{0}(x)$, then $(x-\lambda)^{-1}$ exists by Theorem 1 , and hence, it suffices to show that $\|(x-\lambda) y\| \geqq \delta\|y\|$ for all $y \in \mathcal{A}$, where $\delta=d\left[\lambda, W_{0}(x)\right]$.

For $y \in \mathcal{A}$ of unit norm, choose $g \in \mathcal{A}^{*}$, with $\|g\|=1=g(y)$. Let $f(u)$ $=g(u y)$ for $u \in \mathcal{A}$. Clearly, $f \in \mathfrak{p}$, and $\delta\|y\| \leqq|\lambda-f(x)|=|f(\lambda-x)|$ $=|g((\lambda-x) y)| \leqq\|(\lambda-x) y\|$.

THEOREM 2. If $K$ is a closed convex subset of the plane, then $K \supset W_{0}(x)$ if and only if $\left\|(x-\lambda)^{-1}\right\| \leqq d[\lambda, K]^{-1}$ for $\lambda \notin K$.

ProOF. If $K \supset W_{0}(x)$, then Lemma 1 implies that

$$
\left\|(x-\lambda)^{-1}\right\| \leqq d\left[\lambda, W_{0}(x)\right]^{-1} \leqq d[\lambda, K]^{-1} \quad \text { for } \quad \lambda \notin K .
$$

Conversely, suppose that the resolvent of $x$ satisfies the indicated growth condition. To show that $W_{0}(x) \subset K$, it suffices to show that every half-plane $H$ which contains $K$ also contains $W_{0}(x)$. By a preliminary translation and rotation, we may suppose that $H$ is the right half-plane, $\operatorname{Re} z \geqq 0$. Since $H \supset K,\left\|(1+t x)^{-1}\right\|=t^{-1}\left\|\left(t^{-1}+x\right)^{-1}\right\| \leqq 1$ for all $t>0$. Hence, if $f \in \mathfrak{p}$, then $\operatorname{Re} f\left((1+t x)^{-1}\right) \leqq\|f\|\left\|(1+t x)^{-1}\right\| \leqq f(1)$, and thus, $0 \leqq \operatorname{Re} f\left(1-(1+t x)^{-1}\right)$ $=\operatorname{Re} f\left(t x(1+t x)^{-1}\right)$. Dividing by $t$ and letting $t \rightarrow 0$ yields $\operatorname{Re} f(x) \geqq 0$. Since $f$ is arbitrary, this shows that $W_{0}(x) \subset H$.

REMARK. A glance at the proof of Theorem 2 shows that the same conclusion follows from the waker condition 


$$
\left\|(x-\lambda)^{-1}\right\| \leqq d[\lambda, K]^{-1}+o(1) \text { as }|\lambda| \rightarrow \infty .
$$

(See [10] for a special case of this.)

THEOREM 3. $\left|W_{0}(x)\right| \leqq\|x\| \leqq 4\left|W_{0}(x)\right|$.

PROOF. The first inequality is clear. To prove the second, it suffices to show that if $W_{0}(x)$ is a subset of the closed unit disk, then $\|x\| \leqq 4$. Now, by Theorem 2, $\left\|(x-\lambda)^{-1}\right\| \leqq(|\lambda|-1)^{-1}$ for $|\lambda|>1$, and thus, the Cauchy formula

$$
x=\frac{1}{2 \pi i} \int_{|\lambda|=2} \lambda(\lambda-x)^{-1} d \lambda
$$

yields $\|x\| \leqq 4$

Theorem 3 shows that the numerical radius furnishes an equivalent norm on $\mathcal{A}$. The next theorem indicates how the numerical range itself, may be recovered from the given norm on $\mathcal{A}$. We need the following lemma.

LEMMA 2 (Lumer). $\lim _{t \rightarrow \infty}\|x+t\|-t=\sup \operatorname{Re} W_{0}(x)$.

PROOF. If $f \in \mathfrak{p}$, then $\|x+t\| \geqq \operatorname{Re} f(x+t)=t+\operatorname{Re} f(x)$, so that, $\|x+t\|-t$ $\geqq \sup \operatorname{Re} W_{0}(x)$. On the other hand,

$$
\begin{aligned}
\|x+t\| & =\left\|\left(x^{2}-t^{2}\right)(x-t)^{-1}\right\| \leqq t^{2}\left\|(x-t)^{-1}\right\|+\left\|x^{2}\right\|\left\|(x-t)^{-1}\right\| \\
& \leqq t^{2} d\left[t, W_{0}(x)\right]^{-1}+o(1) \leqq t^{2}\left[t-\sup \operatorname{Re} W_{0}(x)\right]^{-1}+o(1)
\end{aligned}
$$

Thus, $\lim _{t \rightarrow \infty} \sup \|x+t\|-t \leqq \sup \operatorname{Re} W_{0}(x)$, which completes the proof.

THEOREM 4. Let $p$ be a complex number. Then, $p \in W_{0}(x)$ if and only if $|p-\lambda| \leqq\|x-\lambda\|$ for all complex $\lambda$. Hence,

$$
W_{0}(x)=\bigcap_{\lambda}\{z:|z-\lambda| \leqq\|x-\lambda\|\} .
$$

ProOF. If $p=f(x) \in W_{0}(x)$, then $|p-\lambda|=|f(x-\lambda)| \leqq\|x-\lambda\|$, for any complex $\lambda$. Conversely, suppose $p \notin W_{0}(x)$. We claim that there is a complex number $\lambda$, such that $|p-\lambda|>\|x-\lambda\|$. In fact, by the convexity of $W_{0}(x)$, there is no loss of generality in assuming that $W_{0}(x)$ lies in the half-plane $\operatorname{Re} z \leqq 0$, and that $p>0$. Hence, Lemma 2 implies $\|x+t\|-t<p$ for large positive $t$.

Lemma 2 yields a formula for the numerical radius : 


$$
\left|W_{0}(x)\right|=\max _{\theta} \lim _{t \rightarrow \infty}\left(\left\|x+t e^{i \theta}\right\|-t\right) .
$$

There is an analogous expression for the spectral radius which follows. This formula appears in [5] (and probably occurs elsewhere in the literature). The proof included here seems to represent a substantial simplification.

THEOREM 5. For $x \in \mathcal{A}$

$$
\lim _{t \rightarrow \infty} \frac{\log \left\|e^{t x}\right\|}{t}=\max \operatorname{Re} \sigma(x)
$$

Thus,

$$
|\sigma(x)|=\max _{\theta} \lim _{t \rightarrow \infty} \frac{\log \left\|\exp \left(t e^{i \theta} x\right)\right\|}{t}
$$

PROOF. The function $\phi(t)=\log \|\exp (t x)\|$ is sub-additive, and thus, $\lim _{t \rightarrow \infty} \phi(t) / t$ exists (see [11], page 17). Therefore, $\lim _{t \rightarrow \infty} \phi(t) / t=\log \lim _{n \rightarrow \infty}\|\exp (n x)\|^{1 / n}$ $=\log \max \{|\lambda|: \lambda \in \sigma(\exp (x))\}=\log \max \{|\exp (\lambda)|: \lambda \in \sigma(x)\}=\max \operatorname{Re} \sigma(x)$. The last statement of the theorem is clear.

2. We turn now to the study of the numerical range in the algebra $\mathscr{B}(X)$ of bounded linear operators on a Banach space $X$. Recall that $X$ may be regarded as a semi-inner-product space by choosing a function $x \rightarrow x^{*}$ from $X$ into $X^{*}$ with the properties $\left\|x^{*}\right\|=\|x\|,\left\langle x, x^{*}>=\|x\|^{2}\right.$ for $x \in X$.

For $T \in \mathscr{E}(x)$, Lumer has defined the numerical range of $T$ as the set $W(T)=\left\{<T x, x^{*}>:\|x\|=1\right\}$.

THEOREM 6. $W_{0}(T)=C W(T)^{-}=$closure of the convex hull of $W(T)$.

PROOF. This result can be obtained from [7]. We give a simple proof.

If $x$ is a unit vector in $X$, then $T \rightarrow\left\langle T x, x^{*}\right\rangle$ is a positive linear functional on $(X)$, hence, $W(T) \subset W_{0}(T)$. This implies $K=\mathcal{C} W(T)^{-} \subset W_{0}(T)$. To prove the reverse inclusion note that $\|(T-\lambda) x\| \geqq\left|<(T-\lambda) x, x^{*}>\right|$ $=\left|\left\langle T x, x^{*}\right\rangle-\lambda\right| \geqq d[\lambda, K]$, for any unit vector $x$, and hence, $\left\|(T-\lambda)^{-1}\right\|$ $\leqq d[\lambda, K]^{-1}$ for $|\lambda|$ sufficiently large. This, coupled with Theorem 2, shows that $K \supset W_{0}(T)$.

COROLlary. If $X$ is a Hilbert space, then $W_{0}(T)=W(T)^{-}$is the closure of the usual numerical range of $T$.

Let $S_{\alpha}$ denote the sector symmetric with respect to the real axis, with vertex at the origin, and angular opening $\alpha$. 
THEOREM 7. Let $x$ be a quasi-nilpotent element of a Banach algebra A. Let $\left\|(x-z)^{-1}\right\| \leqq K / d_{z}^{n}$, for $z \notin S_{\pi / \alpha}$, where $d_{z}=d\left[z, S_{\pi / \alpha}\right]$, and $0 \leqq \pi / \alpha$ $<2 \pi$. Let $\log \left\|(x-z)^{-1}\right\|=o\left(|z|^{-\alpha}\right)$ as $|z| \rightarrow 0$ for $z \in S_{\beta}, \beta>\pi / \alpha$. Then $x^{n}=0$.

ProOF. Let $S=\left\{S_{\pi / \alpha}-a\right\}$, i.e., $S_{\pi / \alpha}$ translated $a$ units to the left, where $a=|\csc \pi / 2 \alpha|$. This choice of $a$ makes the distance from $S$ to $S_{\pi / \alpha}$ equal to 1. For $\left.\lambda=r \exp ^{\prime} i \theta\right) \notin S, d\left[\lambda^{-1}\right.$, Bdry $\left.S_{\pi / \alpha}\right] \geqq|\lambda|^{-1}|\sin (\theta \pm \pi / 2 \alpha)|$, where the sign must be chosen appropriately. But, $|\lambda||\sin (\theta \pm \pi / 2 \alpha)| \geqq 1$, and hence, $d\left[\lambda^{-1}\right.$, Bdry $\left.S_{\pi / \alpha}\right] \geqq|\lambda|^{-2}$ for $\lambda \notin S$. Thus,

$$
\left\|(1-\lambda x)^{-1}\right\|=|\lambda|^{-1}\left\|\left(x-\lambda^{-1}\right)^{-1}\right\| \leqq K|\lambda|^{2 n-1},
$$

for $\lambda \notin S$.

Let $G(\lambda)=\lambda^{-(2 n-1)}\left[(1-\lambda x)^{-1}-\sum_{k=0}^{2(n-1)} \lambda^{k} x^{k}\right]=(1-\lambda x)^{-1} x^{2 n-1}$. Then, $G(\lambda)$ is an entire function, and $\|G(\lambda)\| \leqq K_{1}$ for $\lambda \notin S$, (and hence for $\lambda \in \operatorname{Bdry} S$ ). Since $\lambda \in S$ implies $\lambda \in S_{\beta}$, for $|\lambda|$ sufficiently large,

$$
\log \|G(\lambda)\| \leqq \log \left[|\lambda|^{-1}\left\|\left(x-\lambda^{-1}\right)^{-1}\right\|\|x\|^{2 n-1}\right]=o\left(|\lambda|^{\alpha}\right)
$$

(as $|\lambda| \rightarrow \infty)$. Thus, $\log \|G(w)\|=\log \|G(\lambda+a)\|$ is $o\left(|w|^{\alpha}\right)$ in $S$, and we may apply the classical Phragmén-Lindelöf Theorem ([4] page 393) to conclude that $G(\lambda)$ is bounded in $S$. This means $G(\lambda)$ is bounded in the entire plane, and hence $G(\lambda)=G(0)=x^{2 n-1}$. Thus, $(z-x)^{-1}=\sum_{k=0}^{2 n-1} z^{-(k+1)} x^{k}$, since $x^{2 n}=0$. But, $\left\|(z-x)^{-1}\right\| \leqq K_{3}|z|^{-n}$, for $z$ on the negative real axis, and so $x^{n}=0$.

COROLLARY. Let $T$ be a quasi-nilpotent operator on a Banach space, with $W(T) \subset S_{\pi / \alpha}$. Let $\log \left\|(T-z I)^{-1}\right\|=o\left(|z|^{-\alpha}\right)$ (as $\left.|z| \rightarrow 0\right)$, for $z \in S_{\beta}$, $\beta>\pi / \alpha$. Then, $T=0$.

This corollary appears in Lumer and Phillips [8]. However, they assume a stronger growth condition on the resolvent in $S_{\beta}$, which can be restated as $\log \left\|(T-z I)^{-1}\right\|=o\left(|z|^{-\delta}\right)$, where $\delta<\alpha$. Our result is sharp in that the growth condition in $S_{\beta}$ can not be waakened from $o\left(|z|^{-\alpha}\right)$ to $O\left(|z|^{-\alpha}\right)$. To see this, consider the Volterra operator $(V f)(x)=\int_{0}^{x} f(t) d t$ on $L^{2}[0,1]$. It is well known that $W(V) \subset S_{\pi}$, and one readily verifies that $\log \left\|(V-z I)^{-1}\right\|$ $=O\left(|z|^{-1}\right)$ for all small $z$. A similar example appears in the paper just cited.

We remark that the sectorial angle $\pi / \alpha$ may be greater than $\pi$ in the corollary, since the numerical range $W(T)$ need not be convex. In the other 
direction, there exist quasi-nilpotent operators in a Hilbert space with numerical range contained in a sector of arbitrarily small angle. See for example Kato [6], page $280-281$.

3. Thus far the numerical range has been studied in algebras without involution. In this final section, we will make a few remarks about the situation where adjoints are present. The paper [1] of Berberian and Orland is relevant to this section.

To begin with, note that if $\mathcal{A}$ is a $B^{*}$ algebra $\left(\left\|x^{*} x\right\|=\|x\|^{2}\right)$, then $\mathfrak{p}$ consists of the normalized positive functionals on $\mathcal{A}$, i.e., $f$ 's such that $f(1)=1, f\left(x^{*} x\right) \geqq 0$.

THEOREM 8. Let $\mathcal{A}$ be a $B^{*}$ algebra with unit. If $x \in \mathcal{A}$ is normal, then $W_{0}(x)=\operatorname{Co}(x)$.

PROOF. If $x \in \mathcal{A}$, is normal, then $\|x\|=|\sigma(x)|=$ spectral radius of $x$. It follows that if $D=\{z:|z-\lambda| \leqq r\}$ is any disk containing $\sigma(x)$, then $\|x-\lambda\|$ $=|\sigma(x-\lambda)| \leqq r$. Hence, $W_{0}(x) \subset D$. Since $\mathcal{C} \sigma^{\prime}(x)$ is the intersection of all such disks containing $\sigma(x)$, it follows that $W_{0}(x) \subset C_{o}(x)$.

It is clear from the definition that $W_{0}$ is invariant under norm-preserving isomorphisms. More generally, if $\nu$ is an algebra homomorphism of norm 1 , from a complex Banach algebra $\mathcal{A}$ with unit into another such algebra $\mathcal{A}^{\prime}$, then $W_{0}(\nu(x)) \subset W_{0}(x)$, for each $x \in \mathcal{A}$.

In the remainder of this paper $\nu$ will denote the canonical homomorphism from the algebra $\mathcal{B}(H)$ of operators on a Hilbert space $H$ onto the Calkin algebra $\mathscr{B}(H) / \mathcal{K}$, where $\mathcal{K}$ is the ideal of compact operators in $H$. Both of these are $B^{*}$ algebras [9]. If $T \in \mathscr{B}(H)$, then by the above remark $W_{0}(\nu(T))$ $\subset W_{0}(T)=W(T)^{-}$.

Wolf [14] calls the spectrum $\sigma(\nu(T))$ of the element $\nu(T)$ of the Calkin algebra, the essential spectrum of $T$. (Different definitions occur in $[2,13,14]$.) It is natural to call the numerical range of this element, the essential numerical range of $T$. The following theorem justifies the terminology.

THEOREM 9. If $T \in \mathscr{B}(H)$, then $W_{0}(\nu(T))=\bigcap_{K} W(T+K)^{-}$, where the intersection is taken over all compact operators $K$.

PROOF. From Theorem 4 and the definition of the norm in the Calkin algebra, we have $p \in W_{0}(\nu(T))$ if and only if $|p-\lambda| \leqq\|T+K-\lambda\|$ for each complex number $\lambda$, and each compact operator $K$. Another application of Theorem 4 shows that this condition amounts to the assertion that $p \in W_{0}(T+K)$ for each $K$. 
COROLLARY 1. The following are equivalent conditions on a selfadjoint operator $A$ :

(1) $0 \in W_{0}(\nu(A))$

(2) $0 \in \mathcal{C}\left(\sigma_{\text {ess }}(A)\right)$,

(3) $A$ is not of the form $K \pm M$ where $K$ is compact and $M \geqq \delta>0$.

Proof. We know that $\sigma_{\text {ess }}(A)=\sigma(\nu(A))$; and the convex hull of the spectrum of the self-adjoint element $\nu(A)$ is $W_{0}(\nu(A))$ by Theorem 8. Hence (1) and (2) are clearly equivalent.

If $A=K+M$, with $K$ compact and $M \geqq \delta>0$, then $W_{0}(\nu(A))=W_{0}(\nu(M))$ $\subset W_{0}(M) \subset[\delta, \infty]$. Hence, (1) implies (3).

If $0 \notin W_{0}(\nu(A))$, then by the theorem there exists a compact operator $K$ with $0 \notin W_{0}(A+K)$. It follows that $0 \notin \operatorname{Re} W_{0}(A+K)=W_{0}(A+\operatorname{Re} K)$, and hence $A+\operatorname{Re} K$ is either positive and invertible or negative and invertible. Thus, (3) implies (1).

As an application of the preceeding ideas, we mention the following simple proofs of recent results, due respectively to P.A. Fillmore [3] and H. Radjavi [12].

COROLlaRY 2. Either of the following conditions on $A=A^{*}$ implies that 0 belongs to the essential numerical range of $A$ :

(1) $A=\operatorname{Re} T$, where $T$ is quasi-nilpotent,

(2) $A=T^{*} T-T T^{*}$ is a self commutator.

ProOF. (1) Since $\nu(T)$ is quasi-nilpotent, $0 \in \sigma(\nu(T)) \subset W_{0}(\nu(T))$. Hence $0 \in \operatorname{Re} W_{0}(\nu(T))=W_{0}(\boldsymbol{\nu}(A))$.

(2) Suppose that $T^{*} T-T T^{*}=A \geqq \delta>0$. Then for any positive linear functional $f$, we have $\left\|T^{*} T\right\| \geqq f\left(T^{*} T\right)=f\left(T T^{*}\right)+f(A) \geqq f\left(T T^{*}\right)+\delta$. This implies that $\left\|T^{*} T\right\| \geqq \sup _{f} f\left(T T^{*}\right)+\delta=\left\|T T^{*}\right\|+\delta$, which contradicts the fact that $\left\|T^{*} T\right\|=\left\|T T^{*}\right\|$. The same proof carried out in the Calkin algebra shows that $T^{*} T-T T^{*}$ cannot be of the form $K+M$, where $M \geqq \delta>0$ and $K$ is compact.

REMARK. The converse implication in (2) is also true as Radjavi [12], shows, but this is much deeper.

\section{REFERENCES}

[1] S. K. BERBERIAN AND G. H. ORLAND, On the closure of the numerical range of an operator, Proc. Amer. Math. Soc., 18(1967), 499-503. 
[2] L. A. CobuRN, Weyl's Theorem for non-normal operators, Michigan Math. J., 13(1966), 285-288.

[3] P. A. Fillmore, On the real part of a quasi-nilpotent operator, a talk presented to the Functional Analysis Seminar, Indiana University, Dec. 15, 1967.

[4] E. Hille, Analytic function theory, Vol. II, Ginn and Company, Boston, 1962.

[5] S. KANTOROVITZ, On the characterization of spectral operators, Trans. Amer. Math. Soc., 111(1964), 152-181.

[6] T. KATO, Perturbation theory for linear operators, Springer-Verlag, New York, 1966.

[ 7 ] G. Lumer, Semi-inner-product spaces, Trans. Amer. Math. Soc., 100(1961), 29-43.

[8] G. Lumer AND R. S. Phillips, Dissipative operators in a Banach space, Pacific J. Math., 11(1961), 679-698.

[ 9 ] M. A. NAIMARK, Normed Rings, (translated from the Russian) P. Noordhooff, Groningen, 1960.

[10] G. ORland, On a class of operators, Proc. Amer. Math. Soc., 15(1964), 75-80.

[11] G. Polya And G. Szego, Aufgaben and Lehrsatze aus der Analysis, Springer-Verlag, Berlin, 1925.

[12] H. RadjAvi, Structure of $A^{*} A-A A^{*}$, J. Math. Mech., 16(1966), 19-26.

[13] M. SCHECHTER, Invariance of the essential spectrum, Bull. Amer. Math. Soc., 71 (1965), 365-367.

[14] F. WOLF, On the invariance of the essential spectrum under a change of boundary conditions of parial differential boundary operators, Indag. Math., 21(1959), 142-147.

DEPARTMENT OF MATHEMATICS

INDIANA UNIVERSITY

BLOOMINGTON, INDIANA, U.S. A. 